PROCEEDINGS OF THE

AMERICAN MATHEMATICAL SOCIETY

Volume 125, Number 1, January 1997, Pages 47-50

S 0002-9939(97)03666-6

\title{
ON $k$-REFLEXIVE REPRESENTATIONS OF ALGEBRAS
}

\author{
K. R. FULLER, W. K. NICHOLSON, AND J. F. WATTERS \\ (Communicated by Ken Goodearl)
}

\begin{abstract}
Module theoretic methods are employed to obtain simple proofs of extensions of two theorems of E. A. Azoff regarding the reflexivity of direct sums of copies of an algebra of operators on a finite dimensional Hilbert space.
\end{abstract}

An algebra of operators on a Hilbert space is said to be reflexive in case no larger algebra of operators on that space has the same lattice of invariant subspaces. (See $[2,3,4,9]$, for example.) The purpose of this note is to reexamine, from an algebraist's point of view, some of the classical results on the topic that are contained in E. A. Azoff's paper [2]. By so doing, we are able to provide easily accessible proofs of generalizations of the main theorems in [2].

In [6], given a pair of rings $R$ and $\Delta$, we defined, for each bimodule $M={ }_{R} M_{\Delta}$,

$$
\operatorname{alglat}\left({ }_{R} M_{\Delta}\right)=\left\{\alpha \in \operatorname{End}\left(M_{\Delta}\right) \mid \alpha L \subseteq L \text { for all }{ }_{R} L \leq{ }_{R} M\right\} .
$$

Thus alglat $\left({ }_{R} M_{\Delta}\right)$ is the ring of $\Delta$-linear mappings on $M$ that preserve the lattice of $R$-submodules of $M$; and, letting $\lambda_{M}(R)$ denote the canonical image of $R$ in $\operatorname{End}\left(M_{\Delta}\right)$, we say that ${ }_{R} M_{\Delta}$ is reflexive in case alglat $\left({ }_{R} M_{\Delta}\right)=\lambda_{M}(R)$.

If $\Delta=K$ is a field and $R$ is a $K$-algebra, we shall simply write alglat $(M)$ for $\operatorname{alglat}\left({ }_{R} M_{K}\right)$ and say that $M$ is a reflexive $R$-module when $\lambda(R)=\operatorname{alglat}(M)$. Our terminology corresponds to that of Halmos [10] as follows:

$$
\operatorname{alglat}(M)=\operatorname{alg}\left(\operatorname{lat}\left(\lambda_{M}(R)\right)\right)
$$

and ${ }_{R} M$ is a reflexive module exactly when $\lambda_{M}(R)$ is a reflexive algebra of operators on the $K$-vector space $M$.

A bimodule ${ }_{R} M_{\Delta}$ is said to be $k$-reflexive if the finite direct sum $M^{(k)}$ of $k$ copies of $M$ is reflexive. We recall here that there is a canonical isomorphism $\operatorname{alglat}\left({ }_{R} M_{\Delta}^{(k)}\right) \cong \operatorname{alg}_{k} \operatorname{lat}\left({ }_{R} M_{\Delta}\right)$ where

$$
\begin{aligned}
& \operatorname{alg}_{k} \operatorname{lat}\left({ }_{R} M_{\Delta}\right)=\left\{\alpha \in \operatorname{End}\left(M_{\Delta}\right) \mid \text { for all } m_{1}, \ldots, m_{k} \text { in } M\right. \text { there is } \\
& \left.\qquad \text { an } r \in R \text { with } \alpha m_{i}=r m_{i}, \quad \text { for } i=1, \ldots, k\right\} .
\end{aligned}
$$

The isomorphism is given by $\alpha \mapsto \alpha \mid M$.

Throughout the remainder of this paper, we assume that $K$ is an arbitrary field, that $R$ and $\Delta$ are finite dimensional $K$-algebras, that $e_{1}, \ldots, e_{m}$ is an orthogonal set of primitive idempotents in $R$ with $1=e_{1}+\cdots+e_{m}$, and that $J=J(R)$ is

Received by the editors July 14, 1995.

1991 Mathematics Subject Classification. Primary 16P10; Secondary 47D15.

This research was supported by NATO Collaborative Research Grant 920159 and NSERC Grant A 8075. 
the radical of $R$. The statements " ${ }_{R} M_{\Delta}$ is a bimodule" and " $M$ is an $R$-module" entail $k m=m k$ for all $k \in K, m \in M$.

If ${ }_{R} M_{\Delta}$ is a bimodule and $k \geq 2$, then for any $\alpha \in \operatorname{alg}_{k} \operatorname{lat}(M), m \in M$ and $\delta \in \Delta$, there is an $r \in R$ with $\alpha m=r m$ and $\alpha(m \delta)=r(m \delta)=(r m) \delta$. Thus we see that $\left.\operatorname{alg}_{k} \operatorname{lat}(M)=\operatorname{alg}_{k} \operatorname{lat}_{R} M_{\Delta}\right)$ whenever $k \geq 2$.

Modifying the notation of [5] and [8], if $S$ is a $K$-space and $U$ and $X$ are $\Delta$ modules, we call a $K$-linear mapping $\lambda: S \rightarrow \operatorname{Hom}_{\Delta}(U, X)$ an $S$-representation over $\Delta$. We denote such a system by ${ }_{S}[U, X]$ and we write $s u=\lambda(s)(u)$. Analogous to $\operatorname{alg}_{k}$ lat we let

$$
\begin{aligned}
\mathcal{A}_{k}\left({ }_{S}[U, X]\right)=\left\{\alpha \in \operatorname{Hom}_{\Delta}(U, X) \mid\right. & \text { for all } u_{1}, \ldots, u_{k} \text { in } U \text { there } \\
& \text { is an } \left.s \in S \text { with } \alpha u_{i}=s u_{i} \text { for } i=1, \ldots, k\right\} .
\end{aligned}
$$

The decomposition lemmas of $[8$, Section 2] have versions in this setting. In particular we have

Lemma 1. Let ${ }_{S}[U, X]$ be an $S$-representation over $\Delta$. If $U=\bigoplus_{i=1}^{n} U_{i}$ and $S=$ $\bigoplus_{i=1}^{n} S_{i}$ with $S_{i} U_{j}=0$ whenever $i \neq j$, then $\mathcal{A}_{k}\left({ }_{S}[U, X]\right) \cong \bigoplus_{i=1}^{n} \mathcal{A}_{k}\left(S_{i}\left[U_{i}, X\right]\right)$ as $K$-spaces, so $\mathcal{A}_{k}\left({ }_{S}[U, X]\right)=\lambda(S)$ if and only if $\mathcal{A}_{k}\left(S_{i}\left[U_{i}, X\right]\right)=\lambda\left(S_{i}\right)$ for $i=$ $1, \ldots, n$.

Proof. Let $\alpha \in \mathcal{A}_{k}\left({ }_{S}[U, X]\right)$ and put $\alpha_{i}=\alpha \mid U_{i}$. Then clearly $\alpha_{i} \in \mathcal{A}_{k}\left(S_{i}\left[U_{i}, X\right]\right)$, and the map $\phi: \quad \alpha \mapsto\left(\alpha_{1}, \ldots, \alpha_{n}\right)$ is an injective $K$-homomorphism. Surjectivity is easily established using $S_{i} U_{j}=0$ for $i \neq j$.

The composition length of a finitely generated module $M$ is denoted $c(M)$. In particular, $c\left(M_{\Delta}\right) \leq \operatorname{dim}\left(M_{K}\right)$. Thus the following theorem extends Azoff's [2, Theorem 5.2], and our algebraic approach yields a very short and accessible proof.

Theorem 2. Let $M$ be a $K$-finite dimensional $R-\Delta$ bimodule with decomposition ${ }_{R} M_{\Delta}=M_{1} \oplus \cdots \oplus M_{d}$ as $R-\Delta$ bimodules, and suppose that $c\left(M_{j \Delta}\right) \leq k+1$ for all $j \in\{1, \ldots, d\}$. If $k \geq 2$, then $M^{(k)}$ is a reflexive $R$-module.

Proof. Since $k \geq 2, \operatorname{alg}_{k} \operatorname{lat}(M)=\operatorname{alg}_{k} \operatorname{lat}\left({ }_{R} M_{\Delta}\right)$. Also we may assume that ${ }_{R} M$ is faithful and that each $M_{j} \neq 0$. Since $1=e_{1}+\cdots+e_{m}$ is a sum of an orthogonal set of primitive idempotents in $R$, according to Lemma 1 we need only show that ${ }_{R e}[e M, M]$ is $k$-reflexive for each primitive idempotent $e \in R$. Since $\operatorname{Soc}\left({ }_{R} M_{j}\right)$ is a $\Delta$-submodule of $M_{j}$ for each $j \in\{1, \ldots, d\}$, there is a $\Delta$-spanning set $\left\{x_{0 j}, x_{1 j}, \ldots, x_{k j}\right\}$ (probably with repetitions) for $e M_{j}$ with $x_{0 j} \in e \operatorname{Soc}\left({ }_{R} M_{j}\right)$ and $x_{1 j} \neq 0$ if $e M_{j} \neq 0$. Let

$$
x_{i}=x_{i 1}+\cdots+x_{i d} \quad \text { for } i=0,1, \ldots, k .
$$

If $\alpha \in \mathcal{A}_{k}\left(\operatorname{Re}_{e}[e M, M]\right)$, then there is an $r e \in R e$ with $\alpha x_{i}=r e x_{i}$ for $i=1, \ldots, k$; and then $\beta=\alpha-r e \in \mathcal{A}_{k}(\operatorname{Re}[e M, M])$. Since $k \geq 2$, there is an se $\in$ Re with

$$
\text { sex }_{0}=\beta x_{0} \quad \text { and } \quad \text { sex } x_{1}=\beta x_{1}=0 \text {. }
$$

Now since $x_{1}=e x_{1} \neq 0$ and $s e x_{1}=0$, it follows that $s e \in J e$, the unique maximal submodule (see [1, Corollary 17.20 and Theorem 27.11]) of Re. (Otherwise $e \in R e=R s e$, contrary to $e x_{1} \neq 0$.) But then since $x_{0} \in \operatorname{Soc}\left({ }_{R} M\right), \operatorname{sex} x_{0}=0$, and so $\beta x_{0}=0$, and hence $\alpha x_{i}=\operatorname{rex}_{i}$, for all $i=0,1, \ldots, k$. Finally, since the sum $M=M_{1} \oplus \cdots \oplus M_{d}$ is direct and $\alpha e M_{j} \subseteq M_{j}$, we see from

$$
\alpha x_{i 1}+\cdots+\alpha x_{i d}=\operatorname{rex}_{i 1}+\cdots+\operatorname{rex}_{i d}
$$


that $\alpha$ and $\lambda(r e)$ agree on the $\Delta$-spanning set $\left\{x_{i j} \mid i=0,1, \ldots, k, j=1, \ldots, d\right\}$ for $e M$.

If $R=\left\{\left[\begin{array}{ll}a & b \\ 0 & a\end{array}\right] \mid a, b \in K\right\}$, then $M=\left[\begin{array}{c}K \\ K\end{array}\right]$ is not reflexive, so Theorem 2 fails for $k=1$, even for commutative algebras.

Taking $d=1$, we have the following corollary extending [2, Theorem 3.1].

Corollary 3. If $n \geq 3$ and $c\left(M_{\Delta}\right) \leq n$, then ${ }_{R} M$ is $n-1$-reflexive.

Azoff's Theorem 4.1 [2] concerns commutative algebras. To deal with it, we need the following two lemmas. The first is part of [6, Corollary 1.9], and the second was remarked in [7]. We include proofs for the sake of completeness.

Lemma 4. Let ${ }_{R} M_{\Delta}=N \oplus L$. If ${ }_{R} N_{\Delta}$ is reflexive and ${ }_{R} N$ generates ${ }_{R} L$, then ${ }_{R} M_{\Delta}$ is reflexive.

Proof. First suppose $f: N \rightarrow L$ is an $R$-homomorphism and $\alpha \in \operatorname{alglat}(M)$. Then for any $n \in N$ there is an $r \in R$ with

$$
r n+f(r n)=\alpha(n+f(n))=\alpha n+\alpha f(n)
$$

so, since the sum is direct, $\alpha f(n)=f(\alpha n)$. Assume now that $N$ is reflexive and generates $L$ over $R$. Then there is an $r \in R$ with $\alpha n=r n$ for all $n \in N$, and for each $l \in L$ there are $f_{i}: N \rightarrow L$ and $n_{i} \in N$ with $\sum_{i=1}^{m} f_{i}\left(n_{i}\right)=l$, so that $\alpha l=\sum_{i=1}^{m} f_{i}\left(\alpha n_{i}\right)=r l$. Thus $\alpha=\lambda(r)$.

If $M$ is a finitely generated left $R$-module, then its dual module $M^{*}=$ $\operatorname{Hom}_{K}(M, K)$ is a finitely generated right $R$-module and ( $)^{*}=\operatorname{Hom}_{K}(, K)$ defines a duality between the categories of finitely generated left and right $R$-modules. The submodules of $M^{*}$ are just those of the form $\left\{\gamma \in M^{*} \mid \gamma(L)=0\right\} \cong(M / L)^{*}$ for the various ${ }_{R} L \leq{ }_{R} M$. Thus it follows that

$$
\operatorname{alglat}\left(M^{*}\right)=\left\{\alpha^{*} \mid \alpha \in \operatorname{alglat}(M)\right\} .
$$

Moreover the action of $r \in R$ on $M^{*}$ is just $\rho_{M^{*}}(r)=\lambda_{M}(r)^{*}$. Thus we have

Lemma 5. If ${ }_{R} M$ is finitely generated, then $M$ is reflexive if and only if $M^{*}$ is reflexive.

If the finite dimensional algebra $R$ is commutative, then $R$ is a direct sum of local rings with identity elements the idempotents $e_{1}, \ldots, e_{m}$, so ${ }_{R} M_{\Delta}$ is reflexive if and only if each of the bimodules $R_{i} e_{i} M_{\Delta}$ is reflexive. (See Lemma 1.) Our final result extends [2, Theorem 4.1] on commutative $\mathbf{C}$-algebras by dealing with an arbitrary field and replacing vector space dimension by the generally smaller composition length $c\left({ }_{R} M\right)$. Letting $\left\lfloor\frac{n}{2}\right\rfloor$ denote the greatest integer $\leq \frac{n}{2}$, we have

Theorem 6. Let $R$ be a finite dimensional commutative $K$-algebra. If $n \geq 4$ and $c\left({ }_{R} M\right) \leq n$, then $M$ is $\left\lfloor\frac{n}{2}\right\rfloor$-reflexive.

Proof. Write $k=\left\lfloor\frac{n}{2}\right\rfloor$ and recall that, since $k \geq 2$, $\operatorname{alg}_{k} \operatorname{lat}\left({ }_{R} M_{R}\right)$. Assume, as we may, that $R$ is local. Let $\alpha \in \operatorname{alg}_{k} \operatorname{lat}(M)$. If ${ }_{R} M$ is semisimple so that $M=$ $R m_{1} \oplus \cdots \oplus R m_{d}$, then $\alpha\left(m_{1}+\cdots+m_{d}\right)=r\left(m_{1}+\cdots+m_{d}\right)$ implies $\alpha=\lambda(r)$, since $\alpha \in \operatorname{alglat}\left({ }_{R} M_{R}\right)$ and the sum is direct. Otherwise, by the Krull-Schmidt Theorem, $M=N \oplus L$ where is $L$ semisimple (or 0 ), and $N \neq 0$ with $\operatorname{Soc}\left({ }_{R} N\right) \subseteq J N$. In this case, $N^{(k)}$ generates $L^{(k)}$ since (to within isomorphism) $R / J$ is the unique simple $R$-module. Thus by Lemma 4 we need only show that $N^{(k)}$ is reflexive. 
If $c(N / J N) \leq k$, then by Nakayama's Lemma, ${ }_{R} N$ is spanned by $k$ elements, so since it consists of $R$-homomorphisms, $\operatorname{alg}_{k} \operatorname{lat}(M)=\lambda(R)$, i.e., $N^{(k)}$ is reflexive. If $c(N / J N)>k$, then $c\left(\operatorname{Soc}\left({ }_{R} N\right)\right) \leq k$. But then, since $N^{*} /\left(N^{*} J\right) \cong \operatorname{Soc}\left({ }_{R} N\right)^{*}$ (see [1, Exercise 23.6]), $N^{*}$ is spanned by $k$ elements, and so $\left(N^{(k)}\right)^{*} \cong\left(N^{*}\right)^{(k)}$ is reflexive and Lemma 5 applies.

In conclusion, we note that Azoff also provided examples in [2] showing that his results are best possible for finite dimensional Hilbert spaces (i.e., finitely generated modules over finite dimensional $\mathbf{C}$-algebras) in terms of vector-space dimension. These examples are actually field independent in the sense that they are valid for a finite dimensional algebra $R$ over any field $K$.

On the other hand, suppose $K$ is algebraically closed. If $R$ is commutative, then $c\left({ }_{R} M\right)=\operatorname{dim}\left(M_{K}\right)$; if ${ }_{R} M$ is indecomposable, then $c\left(M_{\Delta}\right)=\operatorname{dim}\left(M_{K}\right)$. In particular, when $K=\mathbf{C}$ our Theorem 6 does not improve upon Azoff's Theorem 4.1. However, consider the rational algebra $R=\mathbf{Q}[x] /\left(p(x)^{2}\right)$ where $p(x)$ is an irreducible polynomial of degree 3 . Theorem 6 implies that the regular representation ${ }_{R} R_{\mathbf{Q}}$ is 2-reflexive since $c\left({ }_{R} R\right)=2$, but $\operatorname{dim}\left(R_{\mathbf{Q}}\right) / 2=3$.

\section{ACKNowledgement}

The authors wish to thank the Mathematics Department of the University of Hawaii for its hospitality during the preparation of this manuscript.

\section{REFERENCES}

[1] F. W. Anderson and K. R. Fuller, Rings and Categories of Modules, Second Edition, SpringerVerlag, New York, Berlin, etc. 1992. MR 94i:16001

[2] E. A. Azoff, K-reflexivity in finite dimensional spaces, Duke Math. J. 40 (1973), 821-830. MR 48:9415

[3] _ On finite rank operators and preannihilators, Mem. Amer. Math. Soc. 357 (1986). MR 88a:47041

[4] J. A. Deddens and P. A. Fillmore, Reflexive linear transformations, Linear Algebra and Appl. 10 (1975), 89-93. MR 50:10856

[5] L. Ding, Separating vectors and reflexivity, Linear Algebra and Appl. 174 (1992), 37-52. MR 94a:47075

[6] K. R. Fuller, W. K. Nicholson and J. F. Watters, Reflexive bimodules, Canadian J. Math. 41 (1989), 592-611. MR 90g:16019

[7] Universally reflexive algebras, Linear Algebra and Appl. 157 (1991), 195-201. MR 92g:16009

[8] Algebras whose projective modules are reflexive, J. Pure and Appl. Algebra 98 (1995), 135-150. MR 96c: 16008

[9] D. Hadwin, Algebraically reflexive linear transformations, Linear and Multilin. Alg. 14 (1983), 225-233. MR 85e:47003

[10] P. R. Halmos, Reflexive lattices of subspaces, J. London Math. Soc. 4 (1971), 257-263. MR 44:5808

Department of Mathematics, University of Iowa, Iowa City, Iowa 52242

E-mail address: kfuller@math.uiowa.edu

Department of Mathematics and Statistics, University of Calgary, Calgary, AlBerta, CANADA T2N 1N4

E-mail address: wknichol@math.ucalgary.ca

Department of Mathematics and Computer Science, University of Leicester, LeicesTER, ENGLAND LE1 7RH

E-mail address: jfw@leicester.ac.uk 